\title{
Fundamentals and Applications of High-pressure Induction Thermal Plasmas with Coil Current Modulation
}

\author{
Yasunori Tanaka*
}

Faculty of Electrical Engineering and Computer Science, Institute of Science and Engineering, Kanazawa University, Kakuma, Kanazawa 920-1192, Japan

\begin{abstract}
This paper presents a summary of fundamental principles and applications of modulated induction thermal plasmas that have been developed by the author's group. The modulated induction thermal plasma is established using a coil current that is modulated to the order of several hundreds of amperes at a millisecond time scale. Such a large current modulation enables the creation of a large disturbance in high-pressure and high-temperature plasmas. It also enables control of the temperature and radical density in thermal plasmas in a time domain. Examples are described for application of the modulated induction thermal plasma to surface modification. Thermally and chemically nonequilibrium effects are essential in temperature and radical density fields. The latest result is also presented for various applications of modulated induction thermal plasmas. Finally, dynamic behaviors of a recently developed arbitrarywaveform modulated induction thermal plasma are also introduced as a new type of modulated induction thermal plasma.
\end{abstract}

\section{INTRODUCTION}

Inductively coupled thermal plasma (ICTP) is widely used for various material processes such as synthesis of nanopowders, thermal barrier coatings, diamond film deposition, surface modification, etc. [1-9]. Advantages of using thermal plasma include its much higher densities of reactive species or radicals, and high gas temperatures. However, gas temperatures in thermal plasmas are sometimes too high and are difficult to control. To control gas temperatures and densities of reactive atoms/molecules in thermal plasmas, we developed a system of several tens of kilowatt-class pulse-modulated induction thermal plasma (PMITP) [10-17]. This system can modulate the amplitude of the coil current, allowing induction thermal plasmas to be sustained by a rectangular modulation waveform that is altered on a millisecond time scale. The millisecond rectangular modulation in the coil current perturbs the temperature of thermal plasmas remarkably. It can markedly change densities of atoms and molecules in thermal plasmas. Through this periodical perturbation of the thermal plasmas, we can then control their time-averaged value in the time domain $[16,17]$. This modulation also provides nonequilibrium effects even in high-pressure thermal plasmas. On the other hand, some studies have investigated application of the PMITP to materials processing [18-23]. For example, Ohashi et al. applied the Ar- $\mathrm{H}_{2}$ PMITP for hydrogen doping to $\mathrm{ZnO}$. Their results show that $\mathrm{H}$ atoms can be implanted into $\mathrm{ZnO}$ by treatment with an $\mathrm{Ar}-\mathrm{H}_{2}$ PMITP, thereby improving its photoluminescence [19,20]. We have also investigated application of the $\mathrm{Ar}-\mathrm{N}_{2}$ PMITP for surface nitridation processing of materials [21-23].

*Address correspondence to this author at the Faculty of Electrical Engineering and Computer Science, Institute of Science and Engineering, Kanazawa University, Kakuma, Kanazawa 920-1192, Japan; E-mail: tanaka@ec.t.kanazawa-u.ac.jp
As described herein, the author presents fundamental principles and applications of modulated induction thermal plasmas that have been developed by the author's group. First, the system of pulse-modulated induction thermal plasmas is described. Secondly, fundamental dynamic behaviors of PMITP obtained mainly through our experiments are introduced. Experimental results indicate that the millisecond rectangular modulation in the coil current provides a large disturbance in the radiation intensity of the Ar line and also that the modulation can change the Ar excitation temperature from 5000 to $10000 \mathrm{~K}$, especially in Ar- $\mathrm{CO}_{2}$ PMITP [16]. Thirdly, an example is presented in applications of modulated induction thermal plasmas for high-speed surface nitridation processing. Results of this work show that the rectangular modulation of the coil current can provide an increased number of excited $\mathrm{N}$ atoms; simultaneously, the decreased enthalpy flow onto a specimen irradiated by $\mathrm{Ar}-\mathrm{N}_{2}$ PMITP. Both the nitrogen atomic density and the enthalpy flow are crucial for nitridation processing using thermal plasmas. On the other hand, our non-equilibrium calculations imply that the above simultaneous control of the increased nitrogen atomic density and the decreased enthalpy flow are attributable to chemically non-equilibrium effects [24-26]. Some other applications of PMITP to surface modification will be introduced briefly [18-20]. Fourthly, the latest results on application of $\mathrm{Ar}-\mathrm{O}_{2}$ PMITP are presented for nanopowder synthesis. Finally, modulated induction thermal plasmas of a new type, i.e. the arbitrary waveform modulated induction thermal plasma (AMITP) are introduced. The time evolution in Ar excitation temperature in Ar AMITP is described [27]. This system can modulate the amplitude of the coil current to follow a control signal formed not only in a rectangular waveform, but also an externally given arbitrary waveform. Such a modulation of the coil current supports the detailed control of temperature of thermal plasmas. 


\section{CHARACTERISTIC TIMES IN THERMAL PLASMAS}

It is important to understand characteristic times for changing the state in thermal plasmas. We consider characteristic times in centimeter-class Ar thermal plasmas around $5000 \mathrm{~K}$ at atmospheric pressure. Typical characteristic times for thermal conduction, diffusion and convection were estimated using the following simple equations

$$
\begin{aligned}
& t_{\text {cond }}=\frac{\rho C_{p} L^{2}}{\lambda} \\
& t_{\text {diff }}=\frac{L^{2}}{D} \\
& t_{\text {conv }}=\frac{L}{u} \\
& t_{\text {reac }}=\frac{1}{\alpha n}
\end{aligned}
$$

where $t_{\text {cond }}, t_{\mathrm{diff}}, t_{\text {conv }}$, and $t_{\text {cond }}$ are, respectively, the characteristic times for thermal conduction, diffusion, convection and reactions, $L$ is the characteristic length, $\rho$ is the mass density, $C_{\mathrm{p}}$ is the specific heat, $\lambda$ is the thermal conductivity, $D$ is the diffusion coefficient, $u$ is the gas velocity, $\alpha$ is the reaction rate, and $n$ is the number density of the plasma. In this estimation, $u$ of $10-100 \mathrm{~m} / \mathrm{s}$ was used for induction of thermal plasmas [26].

According to Table 1, thermal conduction, diffusion and convection have characteristic times of several milliseconds. Reactions have a wide time range because reactions of various types including ionization, recombination and excitation are present in thermal plasmas. On the other hand, the radio-frequency (rf) cycle time of the coil current is about $10^{-7}-10^{-5} \mathrm{~s}$ because the frequency of $0.1-10 \mathrm{MHz}$ is usually adopted for the coil current sustaining high-pressure thermal plasmas. This rf cycle time is much shorter than the above characteristic times except reactions, which means that plasma is statically established with no macroscopic disturbance by such an rf coil current. The modulation cycle time used for modulated thermal plasmas is on the order of milliseconds. This modulation cycle in milliseconds has a similar order to that of the characteristic times in thermal plasmas. Therefore, such a millisecond modulation of the coil current can markedly perturb even the heavy-particle temperature and gas flow fields and reaction fields. Furthermore, controlling the modulation amplitude of the coil current and the modulation waveform enables us to control the temperature and gas flow fields in a time domain.

Table 1. Characteristic Times in Centimeter-class Thermal Plasmas at $5000 \mathrm{~K}$ at Atmospheric Pressure

\begin{tabular}{|c|c|}
\hline Phenomena & Characteristic Time \\
\hline \hline Thermal conduction & $\sim 10^{-4}-10^{-2} \mathrm{~s}$ \\
\hline Diffusion & $\sim 10^{-4}-10^{-3} \mathrm{~s}$ \\
\hline Convection & $\sim 10^{-4}-10^{-3} \mathrm{~s}$ \\
\hline Reactions & $\sim 10^{-7}-10^{-2} \mathrm{~s}$ \\
\hline
\end{tabular}

\section{FUNDAMENTAL PRINCIPLES OF PULSE- MODULATED INDUCTION THERMAL PLASMAS (PMITP)}

\subsection{Concept of Modulated Thermal Plasmas}

Fig. (1) shows the concept of modulated induction thermal plasmas $[28,29]$. We can see two stable operation of plasmas; cold plasma mode and thermal plasma mode. The cold plasma has a relatively high electron temperature around several $\mathrm{eV}$ and a low gas temperature around $\sim 0.03$ $\mathrm{eV}$ at the same time. The cold plasma is in general sustained at lower pressure although the high pressure non-equilibrium plasma is also paid attention recently. On the other hand, thermal plasmas are established at high pressure and high power density. The thermal plasma, has the electron temperature generally close to the gas temperature around $\sim 1.0 \mathrm{eV}$, and the much high density of radicals. Both the cold plasma and the thermal plasma can be sustained stably, and both can transit to another mode each other. There is a transition phase between these modes which is unstable for sustaining plasmas.

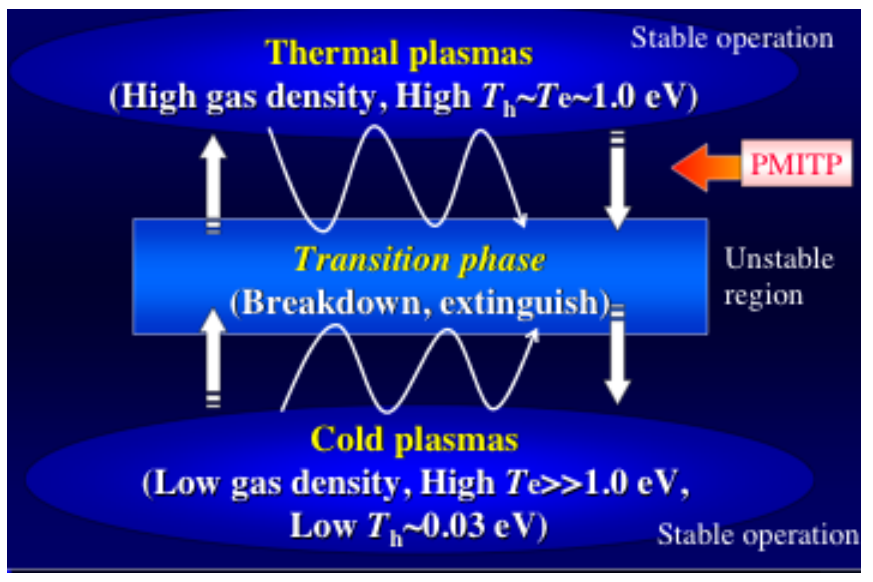

Fig. (1). Concept of pulsation in cold or thermal plasmas.

The pulsing technique was firstly used and has been well utilized for the low-temperature cold plasmas. In this case, the inputted energy or the applied voltage to the cold plasma is rapidly increased to raise the electron temperature and then the radical density, but is rapidly decreased to prevent the cold plasma from arcing or thermal breakdown. On the other hand, the pulsation for thermal plasmas gives control of gas temperature and radical density. The pulsation of the thermal plasma is expected to keep high radical density and to decrease lower gas temperature by utilizing non-equilibrium effects in the transition phase, escaping from the extinction of thermal plasmas.

\subsection{Modulation of Coil Current for PMITP}

For sustaining the pulse modulated induction thermal plasma (PMITP), the coil current modulated in a rectangular waveform shown in Fig. (2) was actually used. For the modulated coil current, the following modulation paramaters are defined: the higher current level (HCL) is the higher amplitude of the coil current, the lower current level (LCL) is the lower amplitude of the coil current, the 'on-time' is the time duration with HCL in a modulation cycle, and the 'offtime' is the time duration with LCL in a modulation cycle. In addition, the shimmer current level (SCL) is also defined as 
a ratio of $\mathrm{LCL} / \mathrm{HCL}$, and the duty factor (DF) is the ratio of 'on-time'/('on-time'+'off-time').

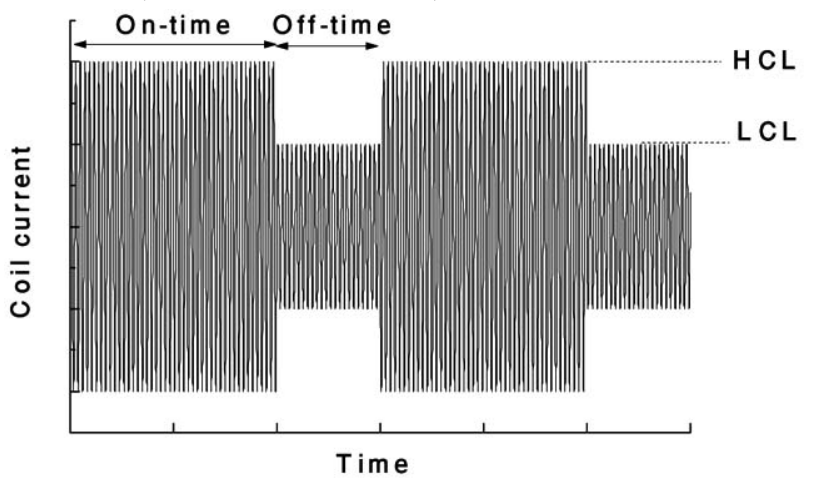

Fig. (2). Pulse modulated coil current.

This modulation of the coil current in milliseconds markedly perturbs thermal plasmas; then the heavy particle temperature of plasmas changes periodically, as described in the previous section. Setting several modulation parameters makes it possible to control the time-averaged temperature of thermal plasmas.

\subsection{Electric Circuit and Plasma Torch}

To realize a PMITP system, we used a high-power semiconductor inverter power supply. Fig. (3) depicts the electric circuit of a power supply for PMITP. The system mainly contains four components: a rectifier circuit, an inverter circuit, an impedance-matching circuit and an ICP torch. Details of this power supply are available in $[16,17]$. The coil current amplitude is modulated by intentionally controlling the fire angle of MOSFET elements to shift from the impedance matching point.

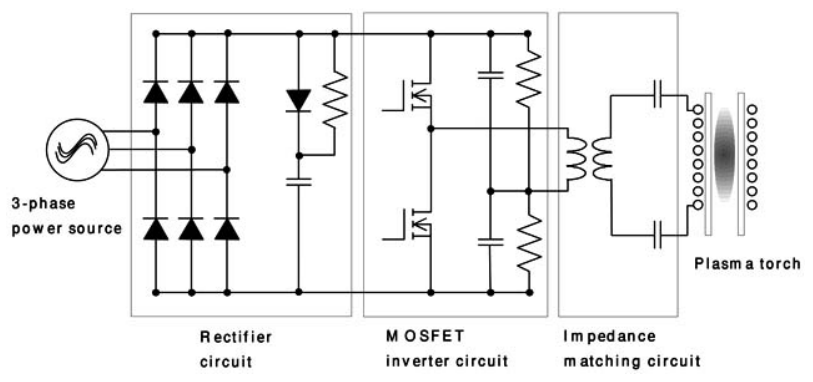

Fig. (3). Electric circuit for PMITP [16, 17].

Fig. (4) presents the configuration of a plasma torch for PMITP. The plasma torch comprises two coaxial quartz tubes with $330 \mathrm{~mm}$ length. The inner tube has $70 \mathrm{~mm}$ internal diameter. Between these two tubes, cooling water flows to maintain the temperature of the tube wall as $300 \mathrm{~K}$. This plasma torch has an eight-turn induction coil around the quartz tube. Downstream of the plasma torch, a water-cooled reaction chamber is installed. Argon and other gas mixtures are supplied as a sheath gas along the inside wall of the inside quartz tube. The dynamic behavior of thermal plasma depends on the configuration and the scale of a plasma torch.

\subsection{Stable Operation Region of PMITP}

The time-averaged input power to the plasma decreases with reducing LCL or with increasing off-time if the HCL

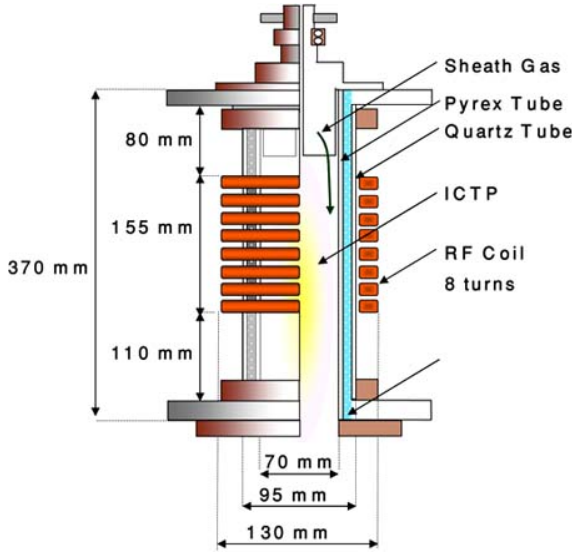

Fig. (4). Schematic diagram of plasma torch.

and on-time are fixed during PMITP operation. Decreasing the input power makes it difficult to sustain the PMITP. Therefore, the stable operation region is limited for the PMITP. Fig. (5) shows the critical boundary of the stable operation region for the PMITP as functions of the SCL and the duty factor DF at atmospheric pressure. This region was measured by changing LCL and off-time with a fixed HCL and a fixed on-time. The HCL was fixed at the value when the input power was $30 \mathrm{~kW}$ for non-modulation operation. The admixture rate of an additional molecular gas to Ar was set to $2.5 \mathrm{~mol} \%$ if the molecular gas is added. The on-time was fixed at $10 \mathrm{~ms}$. As portrayed in Fig. (5), the stable operation region is wide for an Ar PMITP; instability occurs at $\mathrm{SCL}<50 \%$ and $\mathrm{DF}<14 \%$. This stable operation region becomes small for Ar PMITP with an additional molecular gas. Especially, Ar PMITP with $\mathrm{CO}_{2}$ has the smallest stable operation region compared to other molecular gases such as $\mathrm{H}_{2}, \mathrm{O}_{2}$, and $\mathrm{N}_{2}$. For that reason, PMITP is useful for studying the plasma quenching efficiency of molecular gases, which is useful, for example, for circuit-breaker fields.

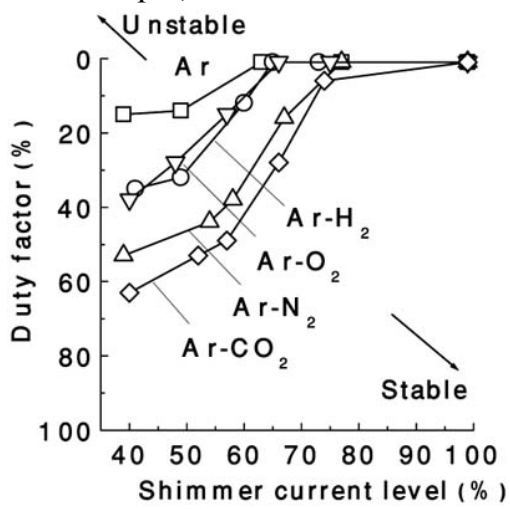

Fig. (5). Stable operation region for different PMITP [16].

\subsection{Dynamic Behaviors of PMITP}

\subsubsection{Response of PMITP}

Fundamental dynamic behavior is a basic datum that is useful to elucidate the nature of PMITP. Fig. (6) (a) portrays the pulse control signal; (b) shows the coil current amplitude for Ar PMITP; (c) shows the coil current amplitude for Ar$\mathrm{N}_{2}$ PMITP; and (d) shows the coil current amplitude for Ar$\mathrm{CO}_{2}$ PMITP [16, 17]. The shimmer current level $(\mathrm{SCL}=\mathrm{LCL} / \mathrm{HCL})$ is taken as a parameter. The additional gas 
admixture ratio is $2.5 \mathrm{~mol} \%$ for $\mathrm{N}_{2}$ and $\mathrm{CO}_{2}$. For any gas mixture case, the coil current amplitude can be changed in 80 $\mu$ s during its rapid increase and its rapid decrease. As seen in this figure, the electric current amplitude depends on the gas composition because the gas composition changes plasma conductance and thus the plasma impedance. Consequently, the inherent characteristic times of thermal plasmas with different gas mixtures can be estimated using this PMITP system, because the inherent characteristic times are much longer than the time shown above for rapid increase in the current amplitude.

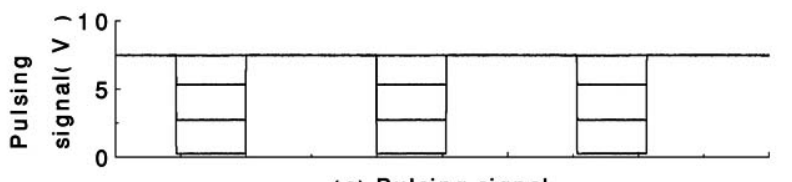

(a) Pulsing signal

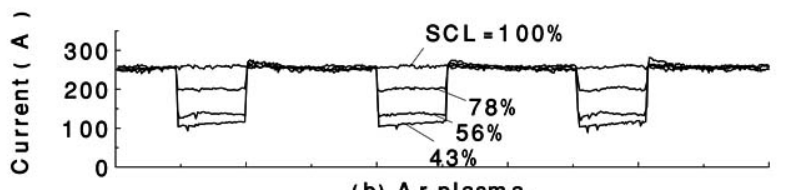

(b) A r plasma.

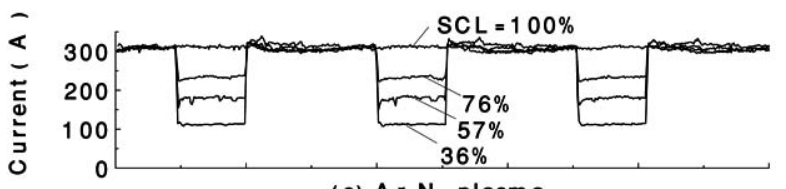

(c) A r-N ${ }_{2}$ plasm a.

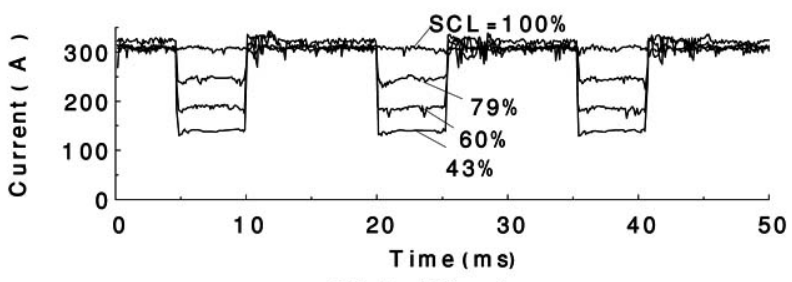

(d) A r- $-\mathrm{CO}_{2}$ plasma.

Fig. (6). Coil current waveform for different PMITPs [16, 17].

Next, some examples of dynamic behaviors of Ar PMITP with or without molecular gases are presented. Fig. (7) portrays the time evolution in radiation intensity of $\mathrm{Ar}$ atomic line at $751 \mathrm{~nm}$ for different SCL $[16,17]$. The input power is $30 \mathrm{~kW}$ for $100 \% \mathrm{SCL}$, i.e. for non-modulation operation, and the pressure is atmospheric pressure in the torch. The Ar gas flow rate is $100 \mathrm{slpm}$; the additional gas flow rate is $2.5 \mathrm{slpm}$. The radiation intensity of the Ar line was measured at $10 \mathrm{~mm}$ below the coil end, where the plasma has higher temperature. As presented in the figure, the radiation intensity can be modulated following the modulated coil current. This change in the radiation intensity means that the number of Ar excited atoms in the PMITP changed according to the coil current modulation. That response in radiation intensity from $\mathrm{Ar}-\mathrm{N}_{2}$ or $\mathrm{Ar}-\mathrm{CO}_{2}$ PMITP is slower than that from $\mathrm{Ar}$ PMITP. This difference in response time indicates that addition of molecular gas makes it difficult for Ar thermal plasmas to recover from a lowtemperature state to a high-temperature state in the PMITP.

\subsubsection{Response of Ar Excitation Temperature in PMITP}

Next, the time evolution in Ar excitation temperature was estimated to confirm effects of such a coil current
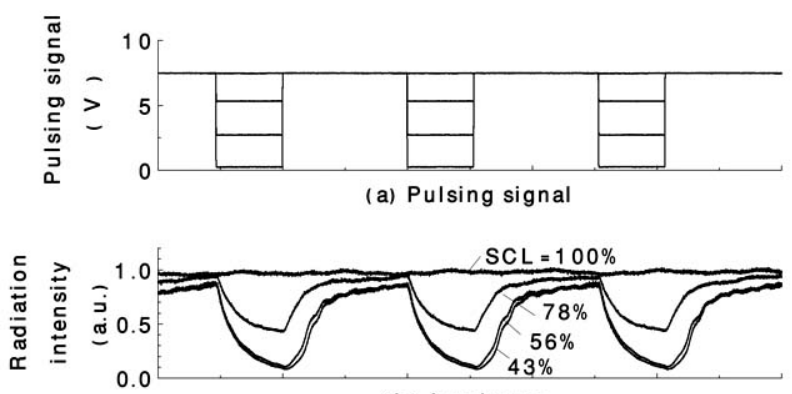

(b) A r plasm a.
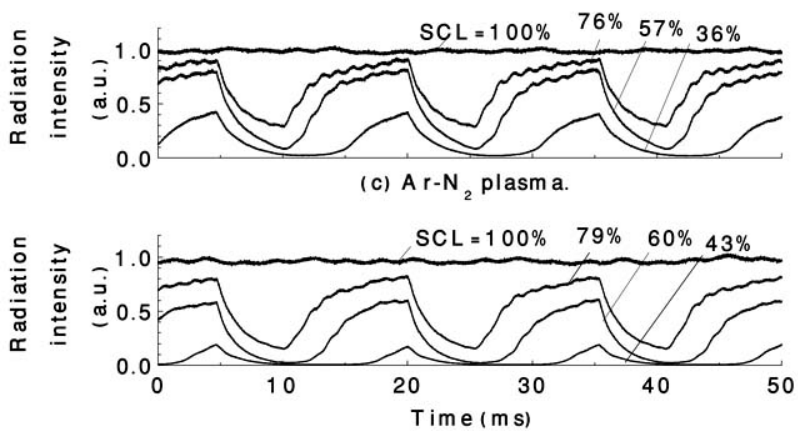

(d) $\mathrm{A} \mathrm{r}-\mathrm{CO}_{2}$ plasma.

Fig. (7). Time evolution in radiation intensity of Ar line at $751 \mathrm{~nm}$ from different PMITPs $[16,17]$.

modulation in the rectangular waveform on the temperature of thermal plasmas $[16,17]$. The input power is $30 \mathrm{~kW}$ for $100 \% \mathrm{SCL}$, i.e. for non-modulation operation. The pressure in the torch is atmospheric pressure. The Ar gas flow rate is $100 \mathrm{slpm}$, and the additional gas flow rate is $2.5 \mathrm{slpm}$. The measurement point is that at $10 \mathrm{~mm}$ below the coil end, which is a high-temperature region in the plasma. The Ar excitation temperature was determined using the two-line method assuming a Boltzmann distribution for the population of excited atoms. The net radiation intensities of Ar atomic lines at $703 \mathrm{~nm}$ and $714 \mathrm{~nm}$ were obtained after subtracting the continuum intensity around $709 \mathrm{~nm}$ from the measured intensities at 703 and $714 \mathrm{~nm}$ for determination of the Ar excitation temperature. Fig. (8) portrays (a) the pulse control signal, (b) the time evolution in the Ar excitation temperature for Ar PMITP, (c) that for $\mathrm{Ar}-\mathrm{N}_{2}$ PMITP, and (d) that for $\mathrm{Ar}-\mathrm{CO}_{2}$ PMITP. These $\mathrm{Ar}$ excitation temperatures were also measured at $10 \mathrm{~mm}$ below the coil end. In the case of Ar PMITP, the Ar excitation temperature is only slightly changed by modulation of the coil current. On the other hand, in molecular gas seeded Ar PMITP, i.e. $\mathrm{Ar}-\mathrm{N}_{2}$ and $\mathrm{Ar}-\mathrm{CO}_{2}$ PMITP, the Ar excitation temperature can be changed markedly following the modulated coil current. Particularly, in $\mathrm{ArCO}_{2}$ PMITP, the coil current modulation can change the Ar excitation temperature by $2000-8000 \mathrm{~K}$ depending on the shimmer current level (SCL).

Fig. (9) presents the maximum and minimum values of the Ar excitation temperature in a modulation cycle in $\mathrm{Ar}$ PMITP with a different molecular gas. As presented in diagram (a), the maximum temperature remains almost unchanged at about $17000 \mathrm{~K}$ as the SCL is varied. On the other hand, the minimum value drops as the SCL is reduced, 


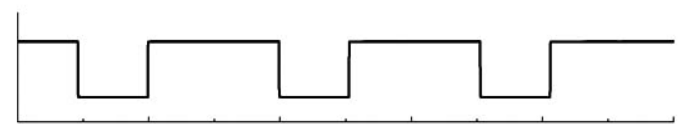

(a) Pulsing signal
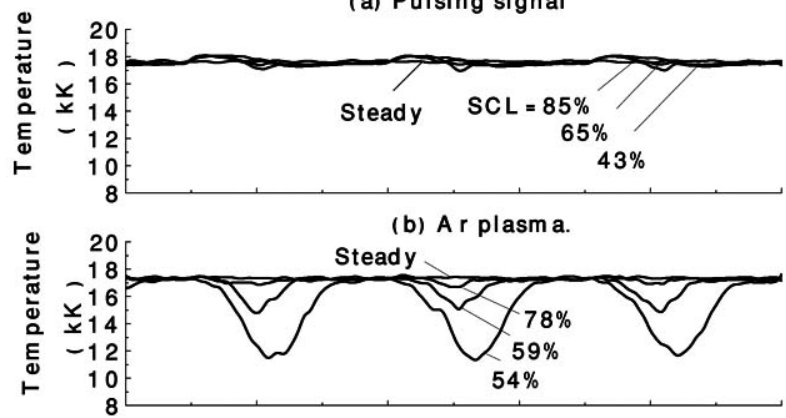

(c) $\mathrm{A} \mathrm{r}-\mathrm{N}_{2}$ plasm a.

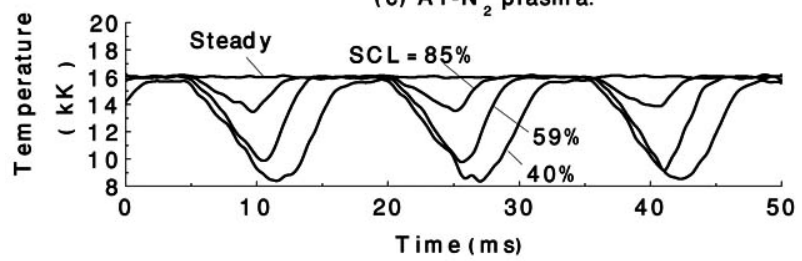

(d) A r-C $\mathrm{CO}_{2}$ plasm a.

Fig. (8). Time variation in Ar excitation temperature in different PMITPs $[16,17]$.

which suggests that one can control only the minimum temperature at a fixed maximum temperature by varying the SCL or LCL at a fixed HCL. In particular, in the case of $\mathrm{CO}_{2}$ inclusion, the minimum temperature drops to $8000 \mathrm{~K}$, which is about half the maximum temperature of $16000 \mathrm{~K}$ at $\mathrm{SCL}=40 \%$. That fact indicates that $\mathrm{CO}_{2}$ produces more efficient cooling of the thermal plasma than the other gases described here.
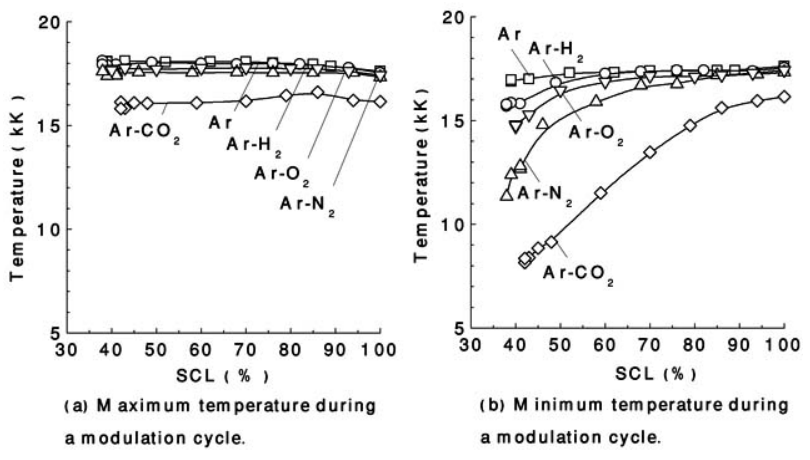

Fig. (9). Maximum and minimum values of $\mathrm{Ar}$ excitation temperature in a modulation cycle in different PMITPs [16].

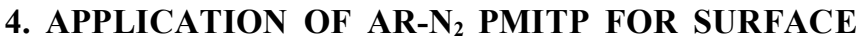 NITRIDATION}

It is interesting to apply PMITP with controlled temperature and radical densities to material processing. To date, application of PMITP for surface modification processing has been attempted $[21,22]$. Here, we introduce nitridation processing of the Ti metallic surface using Ar- $\mathrm{N}_{2}$ PMITP.

\subsection{Plasma Torch, Reaction Chamber, and Specimen Position for Nitridation Processing}

For nitridation processing, a $\mathrm{Ti}$ specimen was irradiated by $\mathrm{Ar}-\mathrm{N}_{2}$ PMITP downstream of the plasma torch. Fig. (10) portrays a schematic diagram of the plasma torch, the reaction chamber, and the titanium specimen for nitridation processing. The plasma torch is identical to that depicted in Fig. (4). Downstream of this plasma torch, a water-cooled reaction chamber with a specimen holder is installed. The specimen holder holds a 15-mm-diameter, 5-mm-thick titanium specimen. The specimen was irradiated directly by an Ar- $\mathrm{N}_{2}$ PMITP. The Ar- $\mathrm{N}_{2}$ gas mixture was supplied as a sheath gas with a swirl along the interior of the inner quartz tube from the top of the plasma torch. The total gas flow rate was fixed at $100.0 \mathrm{slpm}$, where the unit 'slpm' is the standard liters per minute. The nitrogen gas flow rate $Q_{\mathrm{N}_{2}}$ was set to a value of 2.0 or $4.0 \mathrm{slpm}$. Pressure inside the chamber was fixed to $30 \mathrm{kPa}$ (230 Torr). The 'on-time' and 'off-time' were set respectively to $10 \mathrm{~ms}$ and $5 \mathrm{~ms}$ in this experiment. In the present work, we fixed the time-averaged input power to the MOSFET inverter power supply to the same value of $15 \mathrm{~kW}$ for any SCL condition. This fixed power control can be realized using a higher HCL and a lower LCL to the current amplitude in the non-modulation condition, as portrayed in Fig. (11). This fixed power condition enables us to compare results under the same electric input power cost.

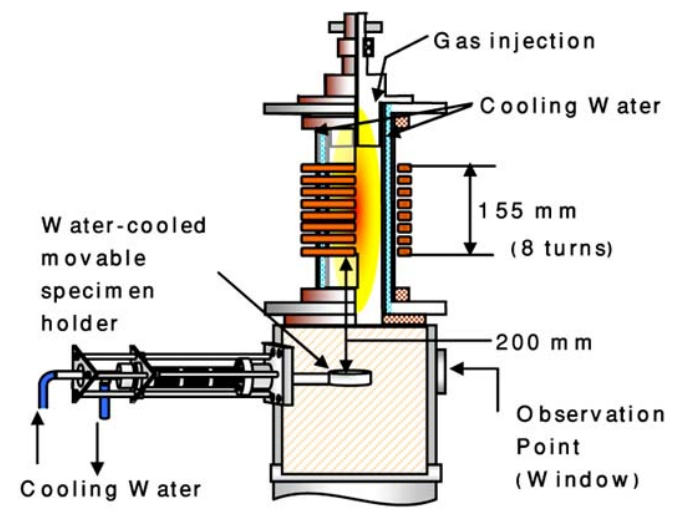

Fig. (10). Specimen position for nitridation processing using PMITP.

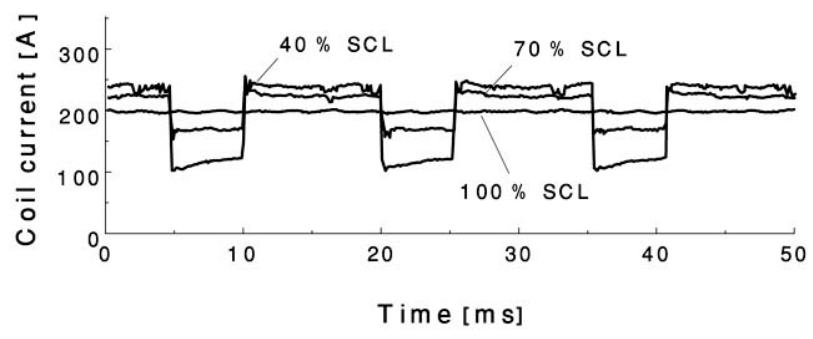

Fig. (11). Coil current amplitude for fixed power operation [22].

Fig. (12) depicts the dynamic behavior of the tail part of the Ar- $\mathrm{N}_{2}$ PMITP, as recorded using a high-speed video camera. The respective gas flow rates of $\mathrm{Ar}$ and $\mathrm{N}_{2}$ are 98 and $2 \mathrm{slpm}$; and the shimmer current level (SCL) is $40 \%$. The time increment of the images in this figure is $1.0 \mathrm{~ms}$. On the other hand, the exposure time for one frame by the highspeed video camera was set to $333 \mu$ s. In panel (2), the plasma tail is visible, extending from the upper side according to "on-time" operation. This plasma tail extends to 
the specimen holder with rotation motion in panels (2)-(5). This rotation motion originates from a supplied swirl gas. The plasma tail goes to the upper side again in panels (5)-(6) in "off-time". These motions are repeated periodically according to coil current modulation.

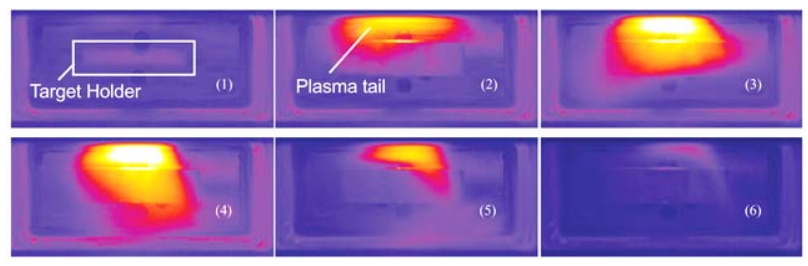

Fig. (12). Dynamic behavior of the plasma tail. The gas flow rate of $\mathrm{Ar} / \mathrm{N}_{2}$ is $98 / 2$ slpm [22].

\subsection{XRD Analysis of the Irradiated Specimen Surface}

The specimen surface of $\mathrm{Ti}$ irradiated by an $\mathrm{Ar}-\mathrm{N}_{2}$ PMITP was analyzed using X-ray diffraction (XRD) to determine the effect of the current modulation on the specimen surface composition. Fig. (13) shows examples of XRD spectra for the specimen surface irradiated by $\mathrm{Ar}-\mathrm{N}_{2}$ PMITP. The Ar and $\mathrm{N}_{2}$ gas flow rates were, respectively, 98 slpm and 2 slpm. In this study, hydrogen is not used, thereby simplifying the experimental condition. The irradiation time is $5 \mathrm{~min}$ for all cases. In general, the nitridation by thermal
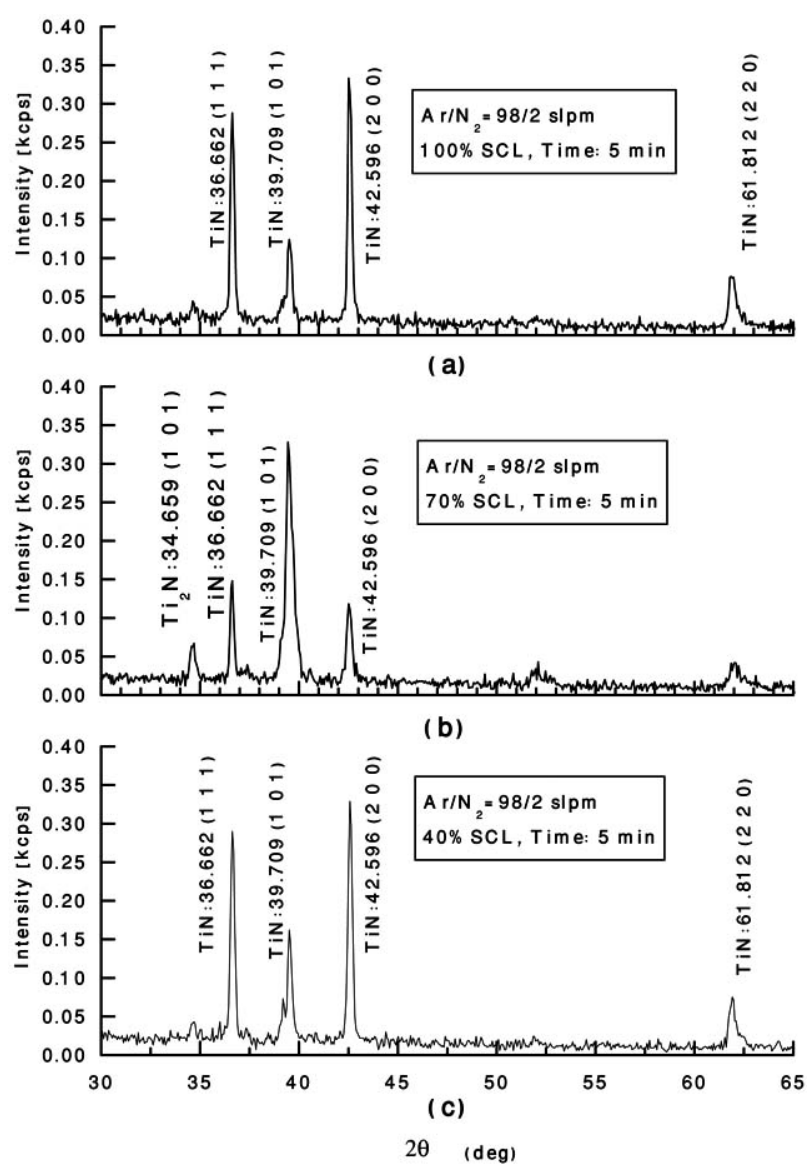

Fig. (13). XRD spectra for specimen surface irradiated by $\mathrm{Ar}-\mathrm{N}_{2}$ PMITP with different shimmer current levels [21, 22]. plasmas depends on the nitrogen atomic flux onto the surface and the surface temperature. In other words, higher nitrogen atomic flux and higher surface temperature promotes surface nitridation of metal. However, higher surface temperature provides higher thermal damage of the surface. As shown there, XRD spectra for TiN ( $\left.\begin{array}{lll}1 & 1 & 1\end{array}\right)$, TiN $\left(\begin{array}{lll}1 & 0 & 1\end{array}\right)$ and TiN $\left(\begin{array}{ll}2 & 0\end{array}\right.$ 0 ) are apparent in the case of $\mathrm{SCL}=100 \%$, i.e. the nonmodulation case. The appearance of these spectra from TiN and no other spectra indicates that the surface nitridation is enough. However, at SCL $=70 \%$, the intensities of $\operatorname{TiN}\left(\begin{array}{ll}1 & 1\end{array}\right.$ 1) and $\operatorname{TiN}\left(\begin{array}{lll}2 & 0 & 0\end{array}\right)$ decrease, whereas those of $\mathrm{TiN}\left(\begin{array}{lll}1 & 0 & 1\end{array}\right)$ increases and the spectrum of $\mathrm{Ti}_{2} \mathrm{~N}\left(\begin{array}{lll}1 & 0 & 1\end{array}\right)$ appears. The appearance of $\mathrm{Ti}_{2} \mathrm{~N}$ indicates that the surface nitridation is not satisfactory. At still lower SCL, i.e. $\mathrm{SCL}=40 \%$, the XRD spectra again come to resemble that at $\mathrm{SCL}=100 \%$, although the modulation condition ( thus the surface temperature ) is different. Consequently, the influence of the coil-current modulation is readily apparent in XRD spectra for the irradiated surface, even at the same input power and at the same specimen position. It is emphasized that the coil current modulation affects the surface structure. As seen in later sections, the coil current modulation increases nitrogen atomic flux and simultaneously decreases the enthalpy flow onto the specimen surface.

\subsection{Modulation Effect on the Increased Number of Nitrogen Excited Atoms}

The neutral nitrogen atomic density irradiating the specimen surface is important for surface nitridation processing using thermal plasmas. We measured the radiation intensity of the nitrogen atomic line at $746.8 \mathrm{~nm}$ to estimate the behavior of the nitrogen atomic density $[21,22]$. The radiation intensity was observed at $200 \mathrm{~mm}$ below the coil end on the axis of the plasma torch. This observation position corresponds to the position just above the specimen surface in case of irradiation experiments. Fig. (14) shows the time evolution in the radiation intensity of the nitrogen atomic line from the Ar- $\mathrm{N}_{2}$ PMITP for SCL of 100, 70, and $40 \%$. The nitrogen gas flow rate $Q_{\mathrm{N}_{2}}$ is $4.0 \mathrm{slpm}$. As shown there, the peak value of the radiation intensity increases with reducing SCL. This result indicates that the pulse modulation of the coil current increases the instantaneous density of the excited nitrogen atom in the observation region, i.e. near the specimen surface.

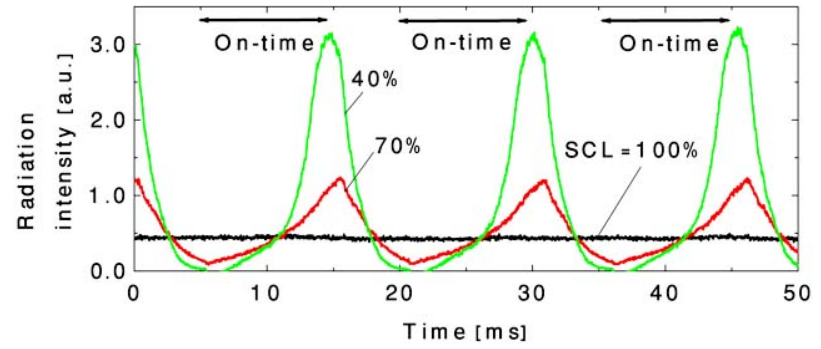

Fig. (14). Time evolutions in the radiation intensity of the nitrogen atomic line at $746 \mathrm{~nm}$ from Ar-N 2 PMITP [21, 22].

For surface nitridation processing, the time-averaged nitrogen density and the instantaneous value are crucial because some nitridation processes require a longer time than the millisecond pulse-modulation cycle. Fig. (15) shows the time-averaged radiation intensity of the nitrogen atomic 
line as a function of SCL for $Q_{\mathrm{N}_{2}}=4.0 \mathrm{slpm}$. As shown there, the time-averaged radiation intensity increases with decreasing SCL, which indicates that the time-averaged nitrogen density also increases with decreasing SCL.

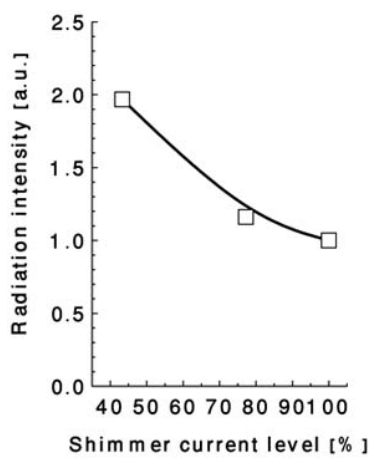

Fig. (15). Time-averaged radiation intensity of the nitrogen atomic line at $746 \mathrm{~nm}$ from $\mathrm{Ar}-\mathrm{N}_{2}$ PMITP [21, 22].

For another on-time condition, we found such a more nitrogen-excited atom in time-averaged value in modulation operation compared with that in non-modulation operation [23]. Fig. (16) presents the time-averaged radiation intensity of the nitrogen atomic line at $746.8 \mathrm{~nm}$, as compared with the intensity in non-modulation operation for different ontimes. Radiation intensity of the nitrogen atomic line at $746.8 \mathrm{~nm}$ for a non-modulated plasma was measured as the input power is increased. The results for the non-modulated plasma are plotted with squares in figure 16 . On the other hand, for a modulated plasma, the time-averaged radiation intensity of the nitrogen atomic line at $746.8 \mathrm{~nm}$ in a modulation cycle was measured with fixed off-time of $5 \mathrm{~ms}$ and fixed HCL and LCL as the on-time is increased. In this case, the input power is also increased by increasing on-time. The results for modulated plasma with $\mathrm{HCL}=385 \mathrm{~A}$ and $\mathrm{LCL}=155 \mathrm{~A}$ are plotted with triangles. Similarly, the results for modulated plasma with $\mathrm{HCL}=355 \mathrm{~A}$ and $\mathrm{LCL}=155 \mathrm{~A}$ are plotted with circles. Note that the horizontal axis in figure 16 is the input power. As shown in this figure, a higher intensity is apparent at $15-22 \mathrm{~kW}$ in modulation operation than that in non-modulation operation.

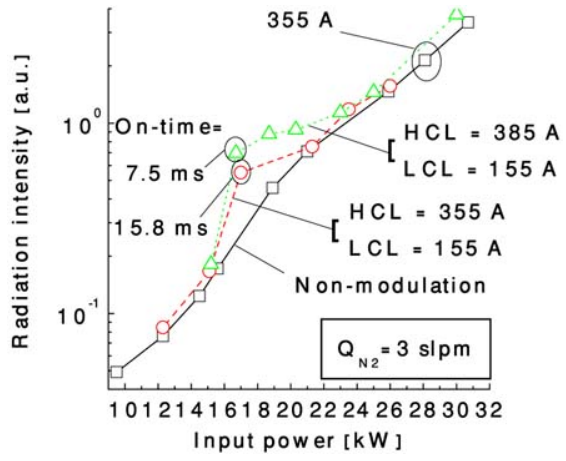

Fig. (16). Time-averaged radiation intensity of the nitrogen atomic line at $746 \mathrm{~nm}$ from $\mathrm{Ar}-\mathrm{N}_{2}$ PMITP for different on-time. Input power is changed by changing the on-time [23].

\subsection{Modulation Effect on the Decreased Specimen Surface Temperature}

The specimen's surface temperature is also a principal factor for nitridation and thermal damage. Fig. (17) depicts the time variation in the surface temperature of titanium specimens for different SCL cases. The Ar- $\mathrm{N}_{2}$ PMITP was irradiated from $t=0 \mathrm{~s}$ in this figure After irradiation of the Ar- $\mathrm{N}_{2}$ PMITP, the surface temperature increases with time. The surface temperature is almost saturated at about $100 \mathrm{~s}$ after irradiation. The irradiation of the $\mathrm{Ar}-\mathrm{N}_{2}$ PMITP was stopped at $t=150 \mathrm{~s}$. After irradiation of the Ar- $\mathrm{N}_{2}$ PMITP, the surface temperature decreases rapidly with time. It is noteworthy that the surface temperature variation depends on the SCL, although the input power to the inverter power supply is the same value of $15 \mathrm{~kW}$ for all cases. From simple analysis of energy balance equation for specimen, results show that the net enthalpy flow on the specimen was decreased by reduction of SCL [22].

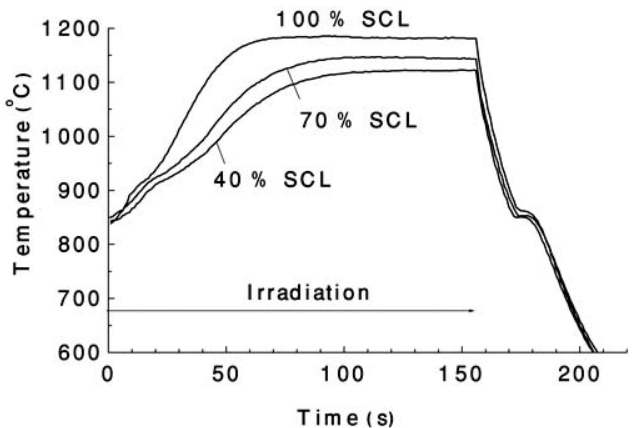

Fig. (17). Temperature variation in surface of specimen irradiated by $\mathrm{Ar}-\mathrm{N}_{2}$ PMITP.

Reducing the SCL increases the excited nitrogen atomic density simultaneously, as described in the previous section. Consequently, reducing SCL, i.e. the modulation of the coilcurrent simultaneously causes both an increase in the excited nitrogen atomic density and a decrease in the net enthalpy flow.

\subsection{Numerical Simulation Considering Non-equilibrium Effects}

The phenomena described above including the increased nitrogen excited atoms and the simultaneously obtained decreased enthalpy flow were unobtainable through analyses of the basis of the local thermodynamic equilibrium condition because more nitrogen excited atoms are obtained by increasing the temperature. To confirm this phenomenon, we developed a two-dimensional, two-temperature chemically non-equilibrium (2D-2TNCE) model of the Ar$\mathrm{N}_{2}$ PMITP [24-26]. This model solves a set of mass conservation equations of a bulk plasma, momentum conservation equations, an energy conservation equation for electrons, an energy conservation equation for heavy particles, a mass conservation equation of each species, and a Maxwell equation for the vector potential with the help of the equation of state and the equation of charge neutrality. The set of the governing equations is presented in Table $\mathbf{2}$.

Fig. (18) shows the time evolution in the spatial distribution of electron temperature $T_{\mathrm{e}}$ and heavy particle temperature $T_{\mathrm{h}}$ in $\mathrm{Ar}-\mathrm{N}_{2}$ PMITP after a rapid increase in the coil current from LCL to HCL. The shimmer current level is $40 \%$ and the $\mathrm{Ar} / \mathrm{N}_{2}$ gas flow rate is $98 / 2 \mathrm{slpm}$. In the reaction chamber, $T_{\mathrm{e}}$ is close to $T_{\mathrm{h}}$. However, in the plasma torch, especially near the torch wall, $T_{\mathrm{e}}$ is apparently increased more rapidly than $T_{\mathrm{h}}$. Therefore, pulse modulation of the coil current thermally enhances non-equilibrium effects near the torch wall. 
Table 2. Governing Equations for a Two-temperature Chemically Non-equilibrium Model.

Mass conservation:

$$
\frac{\partial \rho}{\partial t}+\frac{\partial(\rho u)}{\partial z}+\frac{1}{r} \frac{\partial(r \rho v)}{\partial r}=0
$$

Momentum conservation:

$$
\begin{aligned}
& \frac{\partial(\rho u)}{\partial t}+\frac{\partial(u \rho u)}{\partial z}+\frac{1}{r} \frac{\partial(r v \rho u)}{\partial r} \\
& =\quad-\frac{\partial p}{\partial z}+2 \frac{\partial}{\partial z}\left(\eta \frac{\partial u}{\partial z}\right) \\
& +\quad \frac{1}{r} \frac{\partial}{\partial r}\left[\eta r\left(\frac{\partial u}{\partial r}+\frac{\partial v}{\partial z}\right)\right]+\mu_{0} \sigma \Re\left[\dot{E}_{\theta} \dot{H}_{r}^{*}\right] \\
& \frac{\partial(\rho v)}{\partial t}+\frac{\partial(u \rho v)}{\partial z}+\frac{1}{r} \frac{\partial(r v \rho v)}{\partial r} \\
& =\quad-\frac{\partial p}{\partial r}+\frac{\partial}{\partial z}\left[\eta\left(\frac{\partial v}{\partial z}+\frac{\partial u}{\partial r}\right)\right] \\
& +\quad \frac{2}{r} \frac{\partial}{\partial r}\left(\eta r \frac{\partial v}{\partial r}\right)-2 \eta \frac{v}{r^{2}}+\mu_{0} \sigma \Re\left[\dot{E}_{\theta} \dot{H}_{z}^{*}\right] \\
& \frac{\partial(\rho w)}{\partial t}+\frac{\partial(u \rho w)}{\partial z}+\frac{1}{r} \frac{\partial(r v \rho w)}{\partial r} \\
& =\quad \frac{\partial}{\partial z}\left(\frac{\partial w}{\partial z}\right)+\frac{1}{r} \frac{\partial}{\partial r}\left(r \eta \frac{\partial w}{\partial r}\right)-\frac{\rho v w}{r}-\frac{w}{r} \frac{\partial}{\partial r}(r \eta)
\end{aligned}
$$

Translational energy conservation of heavy particle:

$$
\begin{aligned}
& \frac{\partial\left(\rho h^{\prime}\right)}{\partial t}+\frac{\partial\left(u \rho h^{\prime}\right)}{\partial z}+\frac{1}{r} \frac{\partial\left(r v \rho h^{\prime}\right)}{\partial r} \\
& =\frac{\partial}{\partial z}\left(\lambda_{\mathrm{h}}^{\mathrm{tr}} \frac{\partial T_{\mathrm{h}}}{\partial z}\right)+\frac{1}{r} \frac{\partial}{\partial r}\left(r \lambda_{\mathrm{h}}^{\mathrm{tr}} \frac{\partial T_{\mathrm{h}}}{\partial r}\right) \\
& +\sum_{j(j \neq \mathrm{e})}^{N}\left[\frac{\partial}{\partial z}\left(\rho D_{j}^{\prime} h_{j}^{\prime} \frac{\partial Y_{j}}{\partial z}\right)+\frac{1}{r} \frac{\partial}{\partial r}\left(r \rho D_{j}^{\prime} h_{j}^{\prime} \frac{\partial Y_{j}}{\partial r}\right)\right] \\
& -\quad \sum_{\ell\left(\beta_{e \ell}^{\mathrm{f}} \rho_{\mathrm{e} \ell}^{\mathrm{r}}=0\right)}^{L} \Delta Q_{i}+E_{\mathrm{eh}}
\end{aligned}
$$

Energy conservalion of electron:

$$
\begin{gathered}
\frac{\partial}{\partial t}\left(n_{\mathrm{e}} \frac{5}{2} \kappa T_{\mathrm{e}}\right)+\frac{\partial}{\partial z}\left(u n_{\mathrm{e}} \frac{5}{2} \kappa T_{\mathrm{e}}\right)+\frac{1}{r} \frac{\partial}{\partial r}\left(r n_{\mathrm{e}} \frac{5}{2} \kappa T_{\mathrm{e}}\right) \\
=\frac{\partial}{\partial z}\left(\lambda_{\mathrm{e}}^{\mathrm{tr}} \frac{\partial T_{\mathrm{e}}}{\partial z}\right)+\frac{1}{r} \frac{\partial}{\partial r}\left(r \lambda_{\mathrm{e}}^{\mathrm{tr}} \frac{\partial T_{\mathrm{e}}}{\partial r}\right) \\
-\quad \frac{\partial}{\partial z}\left(\frac{1}{m_{\mathrm{e}}} \frac{5}{2} \kappa T_{\mathrm{e}} \Gamma_{\mathrm{e} z}\right)-\frac{1}{r} \frac{\partial}{\partial r}\left(r \frac{1}{m_{\mathrm{e}}} \frac{5}{2} \kappa T_{\mathrm{e}} \Gamma_{\mathrm{e} r}\right) \\
-\sum_{\ell\left(\rho_{\mathrm{e} \ell}^{\mathrm{f}} \beta_{\mathrm{e} \ell}^{\mathrm{r}} \neq 0\right)}^{L} \Delta Q_{\ell}+\sigma \dot{E}_{\theta} \dot{E}_{\theta}^{*}-P_{\mathrm{rad}}-E_{\mathrm{eh}}
\end{gathered}
$$

Mass conservation of species $j$ :

$$
\begin{aligned}
& \frac{\partial\left(\rho Y_{j}\right)}{\partial t}+\frac{\partial\left(u \rho Y_{j}\right)}{\partial z}+\frac{1}{r} \frac{\partial\left(r v \rho Y_{j}\right)}{\partial r} \\
& =\frac{\partial}{\partial z}\left(\rho D_{j}^{\prime} \frac{\partial Y_{j}}{\partial z}\right)+\frac{1}{r} \frac{\partial}{\partial r}\left(r \rho D_{j}^{\prime} \frac{\partial Y_{j}}{\partial r}\right) \\
& \quad+m_{j} \sum_{i=1}^{L}\left(\beta_{j \ell}^{\mathrm{r}}-\beta_{j \ell}^{\mathrm{f}}\right)\left(\alpha_{i}^{\mathrm{f}} \prod_{i=1}^{N} n_{i}^{\beta_{i \ell}^{\mathrm{f}}}-\alpha_{i}^{\mathrm{r}} \prod_{i=1}^{N} n_{i}^{\beta_{i}^{\mathrm{r}}}\right)
\end{aligned}
$$

Maxwell equation by vector potential:

$$
\begin{aligned}
& \frac{\partial^{2} \dot{A}_{\theta}}{\partial z^{2}}+\frac{1}{r} \frac{\partial}{\partial r}\left(r \frac{\partial \dot{A}_{\theta}}{\partial r}\right)-\frac{\dot{A}_{\theta}}{r^{2}}=\bar{i} \mu_{0} \sigma \omega \dot{A}_{\theta} \\
& \dot{H}_{z_{r}}=\frac{1}{\mu_{0}} \frac{1}{r} \frac{\partial}{\partial r}\left(r \dot{A}_{\theta}\right), \quad \dot{H}_{r}=-\frac{1}{\mu_{0}} \frac{\partial \dot{A}_{\theta}}{\partial z} \\
& \dot{E}_{\theta}=-\bar{i} \omega \dot{A}_{\theta}
\end{aligned}
$$

Reaction heat per unit volume and time:

$$
\Delta Q_{\varepsilon}=\psi_{\text {reac } \varepsilon}\left(\alpha_{\ell}^{\mathrm{f}} \prod_{i=1}^{N} n_{i}^{\beta_{i \ell}^{\mathrm{f}}}-\alpha_{i}^{\mathrm{r}} \prod_{i=1}^{N} n_{i}^{\beta_{i \ell}^{\mathrm{r}}}\right)
$$

Translational enthalpy of heavy particles:

$$
h^{\prime}=\sum_{j \neq \mathrm{e}} Y_{j} h_{j}^{\prime}, \quad h_{j}^{\prime}=\frac{1}{m_{j}}\left(\frac{5}{2} k T_{\mathrm{b}}\right)
$$

Energy transfer from electron to heavy particles:

$$
\begin{aligned}
& E_{\mathrm{eh}}=\sum_{j \neq \mathrm{e}} \frac{3}{2} k\left(T_{\mathrm{e}}-T_{\mathrm{h}}\right) \frac{2 m_{j} m_{\mathrm{e}}}{\left(m_{j}+m_{\mathrm{e}}\right)^{2}} n_{j} n_{\mathrm{e}} \pi \bar{\Omega}_{\mathrm{e} j} \bar{v}_{\mathrm{e} j} \\
& \bar{v}_{\mathrm{e} j}=\sqrt{\frac{8 k T_{\mathrm{e}}\left(m_{\mathrm{e}}+m_{j}\right)}{\pi m_{\mathrm{e}} m_{j}}}
\end{aligned}
$$

Relation between mass density and mass fraction:

$$
n_{j}=\frac{\rho Y_{j}}{m_{j}}
$$

Equation of state:

$$
\rho=\frac{p}{\frac{Y_{\mathrm{e}}}{m_{\mathrm{e}}} K T_{\mathrm{e}}+\sum_{j \neq \mathrm{e}} \frac{Y_{j}}{m_{j}} K T_{\mathrm{b}}}
$$

Quasi-neutrality:

$$
\frac{Y_{\mathrm{e}}}{m_{\mathrm{e}}}=\sum_{j \text { (positive-ion) }} \frac{Y_{j}}{m_{j}}
$$

Balance of mass fraction:

$$
\sum_{j} Y_{j}=1
$$

Effective diffusion coefficient of heavy particle:

$$
D_{j}^{\prime}=\frac{1-Y_{j}}{\sum_{k \neq j} \frac{x_{i} p \Delta_{i j}^{(1)}}{k T_{\mathrm{h}}}}
$$

Electrical conductivity:

$$
\sigma=\frac{e^{2}}{\kappa T_{\mathrm{e}}} \frac{n_{\mathrm{e}}}{\sum_{j \neq \mathrm{e}} n n_{j} \Delta_{\mathrm{e} j}^{(1)}}
$$

Viscosity:

$$
\eta=\sum_{j=1}^{N} \frac{m_{j} n_{j}}{\sum_{i=1}^{N} n_{i} \Delta_{i j}^{(2)}}
$$

Translational thermal conductivity of heavy particle:

$$
\lambda_{\mathrm{h}}^{\mathrm{tr}}=\frac{15}{4} \kappa \sum_{i \neq \mathrm{e}} \frac{n_{i}}{\sum_{j} \xi_{i j} n_{j} \Delta_{i j}^{(2)}}
$$

Translational thermal conductivity of electron:

$$
\begin{aligned}
& \lambda_{\mathrm{e}}^{\mathrm{tr}}=\frac{15}{4} \kappa \frac{n_{\mathrm{e}}}{\sum_{j} \xi_{\mathrm{e} j} n_{j} \Delta_{\mathrm{e} j}^{(2)}} \\
& \frac{1}{\Delta_{i j}^{(1)}}=\frac{3}{8} \sqrt{\frac{\pi \kappa T_{\mathrm{h}, \mathrm{e}}\left(m_{i}+m_{j}\right)}{2 m_{i} m_{j}}} \frac{1}{\pi \bar{\Omega}_{i j}^{(1,1)}} \\
& \frac{1}{\Delta_{i j}^{(2)}}=\frac{5}{16} \sqrt{\frac{\pi K T_{\mathrm{h}, \mathrm{e}}\left(m_{i}+m_{j}\right)}{2 m_{i} m_{j}}} \frac{1}{\pi \bar{\Omega}_{i j}^{(2,2)}} \\
& \xi_{i j}=1+\frac{\left(1-m_{i} / m_{j}\right)\left(0.45-2.54 m_{i} / m_{j}\right)}{\left(1+m_{i} / m_{j}\right)^{2}}
\end{aligned}
$$

where $t$ : lime (s), $r$ : radial position (m), z: axial position (m), $u$ : axial flow velocily $(\mathrm{m} / \mathrm{s}), v$ : radial flow velocily $(\mathrm{m} / \mathrm{s}), \rho$ : mass densily $\left(\mathrm{kg} / \mathrm{m}^{3}\right), p$ : pressure $(\mathrm{Pa}), \eta$ : viscosity $(\mathrm{Pa} \cdot \mathrm{s}), h^{\prime}$ : whole enthalpy of heavy particle $(\mathrm{J} / \mathrm{kg}), h_{j}^{\prime}$ : enthalpy of species $j(\mathrm{~J} / \mathrm{kg}), T_{\mathrm{h}}$ : heavy particle temperature $(\mathrm{K}), T_{\mathrm{e}}$ : electron temperature $(\mathrm{K}), \lambda_{\mathrm{h}}^{\mathrm{tr}}, \lambda_{\mathrm{e}}^{\mathrm{tr}}$ : translational thermal conductivity of heavy particle and electron, respectivly $(\mathrm{W} / \mathrm{m} / \mathrm{K}), \sigma$ : electrical conductivity $(\mathrm{S} / \mathrm{m}), P_{\mathrm{rad}}$ : radiation loss $\left(\mathrm{W} / \mathrm{m}^{3}\right), D_{j}^{\prime}$ : effective diffusion coefficient of heavy particle $j\left(\mathrm{~m}^{2} / \mathrm{s}\right), n_{j}$ : number density of species $j\left(\mathrm{~m}^{-3}\right), Y_{j}$ : mass fraction of species $j, x_{j}$ : mole fraction of species $j, m_{j}$ : mass of species $j(\mathrm{~kg}), \Gamma_{\mathrm{er}}, \Gamma_{\mathrm{ez}}$ : radial and axial electron flux due to ambipoler diffusion $\left(1 / \mathrm{m}^{2} / s\right), \alpha_{\ell}^{\mathrm{f}}, \alpha_{\ell}^{\mathrm{r}}$ : rate coefficients of forward and backward reaction $\ell$, respectively, $\beta_{j \ell}^{\mathrm{f}}, \beta_{j \ell}^{\mathrm{r}}$ : stoichiometric number of species $j$ in forward and backward reaction $\ell, \mu_{0}$ : permeability of vacuum $(\mathrm{H} / \mathrm{m}), \kappa$ : Boltzmann constant $(\mathrm{J} / \mathrm{K}), \dot{A}_{\theta}$ : vector potential $(\mathrm{V} \cdot \mathrm{s} / \mathrm{m}), \omega$ : angular frequency of coil current $(\mathrm{rad} / \mathrm{s}), \dot{E}_{\theta}$ : electric field strength $(\mathrm{V} / \mathrm{m}), \dot{H}_{z}$ : axial magnetic field strength $(\mathrm{A} / \mathrm{m}), \dot{H}_{r}$ : radial magnelic field strenglh $(\mathrm{A} / \mathrm{m}), \bar{i}$ : complex index $\left(\bar{i}^{2}=-1\right), \psi_{\text {reac } \ell}$ : reaclion heal for reaclion $\ell(\mathrm{J}), \Delta Q_{\ell}$ : reaction heal per unil volume and lime $\left(\mathrm{W} / \mathrm{m}^{3}\right)$, $\pi \bar{\Omega}_{i j}^{(1,1)}$ : momentum transfer collision integrals $\left(\mathrm{m}^{2}\right), \pi \bar{\Omega}_{i j}^{(2,2)}$ : viscosity collision integrals $\left(\mathrm{m}^{2}\right), \bar{v}_{\mathrm{e} j}$ : relative average velocity between electron and heavy particles $(\mathrm{m} / \mathrm{s}), N$ : total number of species, $L$ : total number of reactions. $E_{\mathrm{e} h}$ : energy transfer between electron and heavy particles $\left(\mathrm{W} / \mathrm{m}^{3}\right) . R[\dot{M}]$ indicates the real part of a complex $\dot{M}$. 


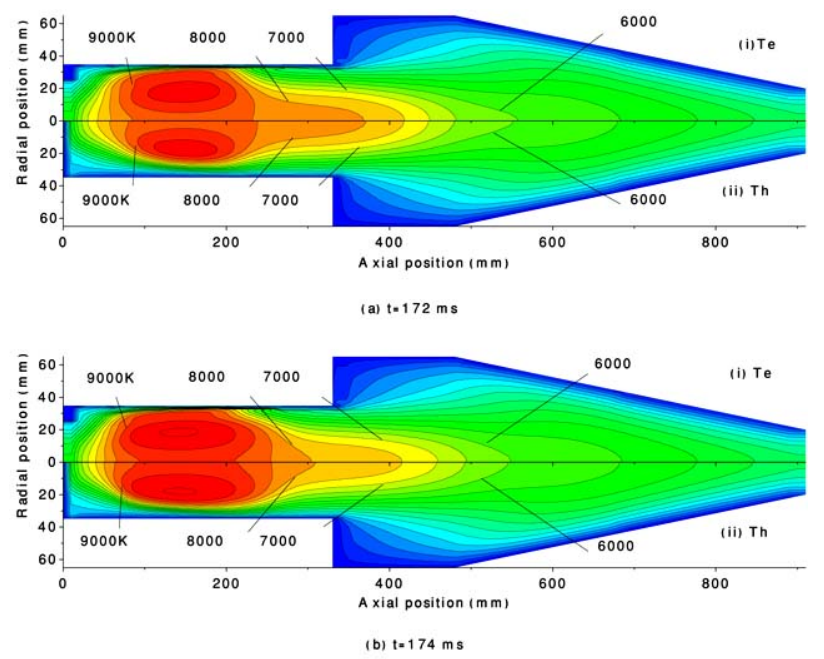

(b) $174 \mathrm{~ms}$

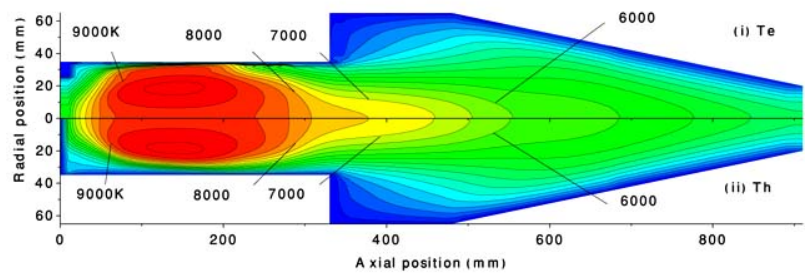

(c) $\mathrm{t}=176 \mathrm{~ms}$.

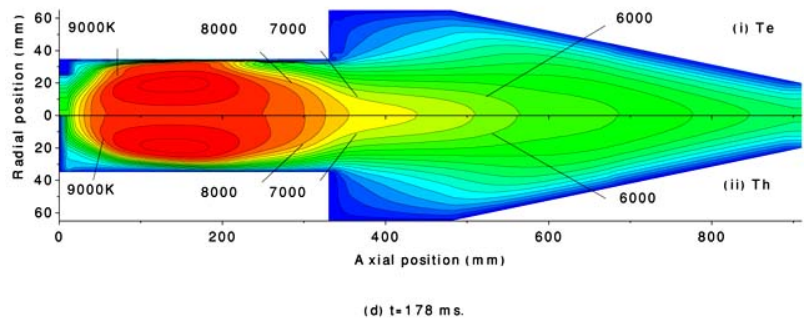

Fig. (18). Transient distribution in electron temperature $T \mathrm{e}$ and heavy particle temperature $T \mathrm{~h}$ in $\mathrm{Ar}-\mathrm{N}_{2}$ PMITP.

Fig. (19) (a) presents the calculated time-averaged mass flow of nitrogen atoms from the Ar- $\mathrm{N}_{2}$ PMITP into the specimen position by decreasing SCL. The nitrogen gas flow rate is $4 \mathrm{slpm}$. As portrayed in this figure, the time-averaged mass flow of nitrogen atoms increases concomitantly with decreasing SCL from $100 \%$ to $40 \%$, meaning that the numerical simulation of $\mathrm{Ar}-\mathrm{N}_{2}$ PMITP also indicates an increase in mass flow of nitrogen atoms onto the specimen surface position. On the other hand, Fig. (19) (b) portrays the calculated time-averaged enthalpy flow from the Ar- $\mathrm{N}_{2}$ PMITP into the specimen surface position. This figure portrays that a reduction of the SCL from $100 \%$ to $40 \%$ decreases the time-averaged enthalpy flow. Consequently, the numerical simulation also supports the fact that the modulation of the coil current produces such an increase in the mass flow of nitrogen atoms and a decrease in enthalpy flow simultaneously, which is also obtained in the experiments. This may be attributed to the following fact: In a modulated plasma, the time-averaged high-temperature region above about $7000 \mathrm{~K}$ in the plasma becomes larger than that in a non-modulated plasma since the hightemperature region is more shrinked in the non-modulated plasma. Thus, more power seems to be comsumed for dissociation of nitrogen molecules in the modulated plasma than in the non-modulated plasma, which produces the higher mass flow of the nitrogen atom and ther lower enthaly flow. However, the effect of the coil current modulation is much greater in the experiment than that predicted by the numerical simulation for increased nitrogen atomic flux. This discrepancy between the experimental and simulation results may be due to the fact that this numerical simulation model considers only 30 reactions in the plasma, neglecting reactions producing nitrogen atom from excited nitrogen molecules, for example.
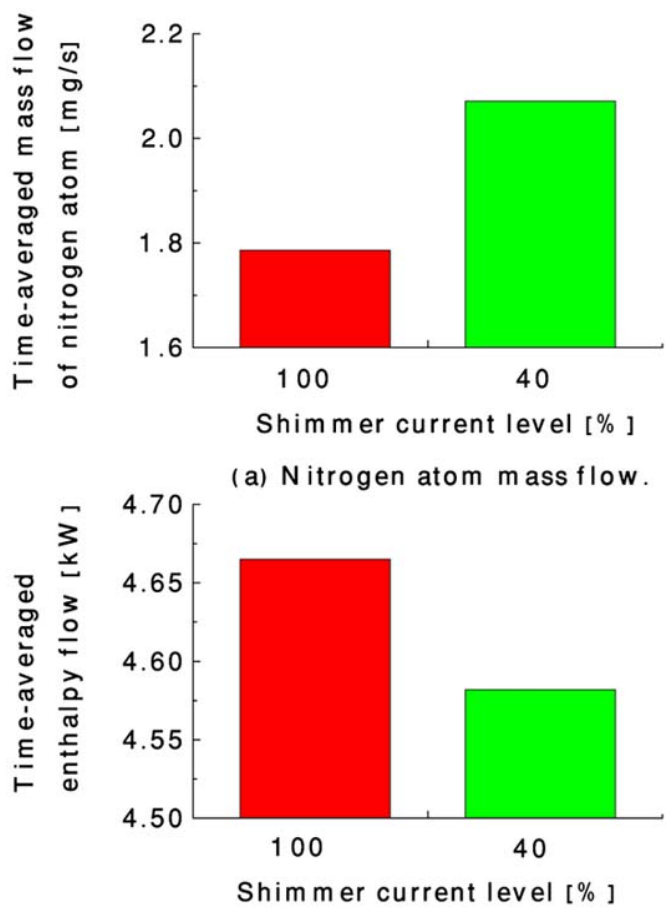

(b) Enthalply flow.

Fig. (19). Calculated time-averaged nitrogen atomic mass flow and enthalpy flow onto the surface position of the specimen.

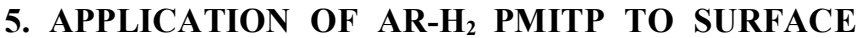 MODIFICATION OF SPECIMENS}

\subsection{Application to $\mathrm{TiO}_{2}$ Specimen [18]}

Ishigaki et al. performed an experiment to apply $\mathrm{Ar}-\mathrm{H}_{2}$ PMITP to surface modification of $\mathrm{TiO}_{2}$. The titanium dioxide $\left(\mathrm{TiO}_{2}\right)$ specimen was irradiated by $\mathrm{Ar}-\mathrm{H}_{2}$ PMITP at $26.6 \mathrm{kPa}$ to obtain effects of PMITP irradiation. The characteristics of $\mathrm{TiO}_{2}$ depend strongly on the formation of lattice defects and the incorporation of hydrogen.

Fig. (20) shows XRD spectra from the $\mathrm{TiO}_{2}$ disk specimen treated in the plasma with and without the coil current modulation at a pressure of $26.6 \mathrm{kPa}$. The parameter in this figure is the specified position, whose details are apparent in Ref. [18]. As the number of the position parameters in this figure increases from 100 to 200 , in millimeter, the distance between the plasma flame and the specimen surface decreases. 
(a) Continuous mode

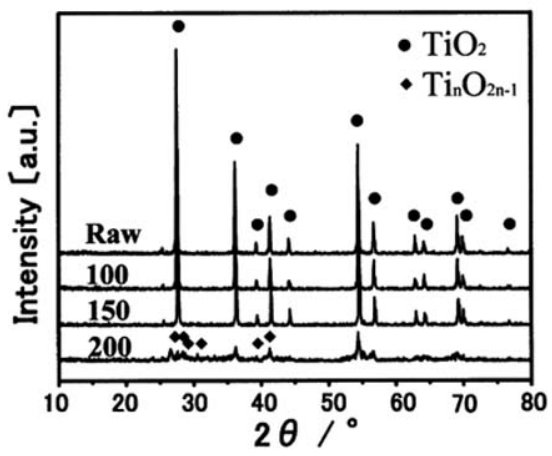

(b) Pulse-modulated mode

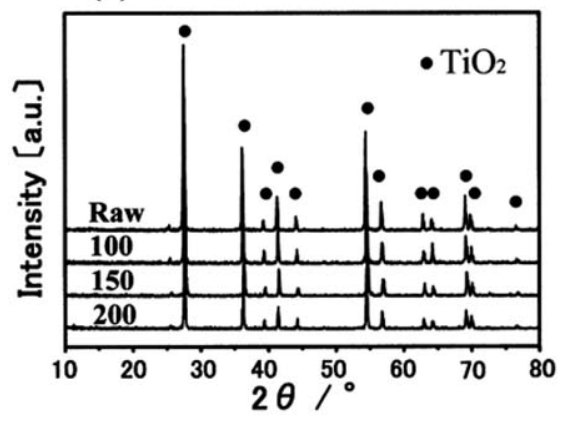

Fig. (20). $\mathrm{XRD}$ spectra from $\mathrm{TiO}_{2}$ disks placed at various positions in $\mathrm{Ar}-\mathrm{H}_{2}$ plasma at a pressure of $26.6 \mathrm{kPa}$ [18].

As this figure shows, the phases of all specimens remain unchanged, except for specimen placed in the plasma of continuous mode at a position parameter of 200. However, Fig. (20) (a) portrays that Magneli phases, $\mathrm{Tin}_{2} \mathrm{O}_{2} n-1$, were formed on the surface of the specimen at a position parameter of 200. The disk specimen, treated at position of 200 under $26 \mathrm{kPa}$ in the continuous plasma, was irradiated directly by the plasma flame; then the surface was heated to a temperature that was higher than that of the remaining specimens. Consequently, the specimen was highly reduced from $\mathrm{TiO}_{2}$ to $\mathrm{Ti}_{n} \mathrm{O}_{2 n-1}$.

However, as presented in Fig. (20) (b), XRD spectra from Magneli phases $\mathrm{Ti}_{2} \mathrm{O}_{2 n-1}$ were not observed. An important point is that the specimens treated in the PMITP were not reduced, even at the highest position of 200, where the specimen temperature is higher than that of the remaining samples and the concentration of hydrogen atoms is presumed to be the highest of all three sample positions. Ishigaki et al. suggested that this difference in the results described above between the irradiation of PMITP and the continuous plasma might be attributed to non-equilibrium effects in the PMITP.

\subsection{Application to H Doping onto a ZnO Specimen $[19,20]$}

Following is an example of adoption of PMITP to hydrogen atom doping to $\mathrm{ZnO}$. Many recent investigations of zinc oxide $(\mathrm{ZnO})$ have reported its quantum effect in superlattices, laser emission, and heterojunction light emitting diode. Consequently, the control of defects related to electron-hole recombination processing is important to improve the $\mathrm{ZnO}$ emission efficiency. Hydrogen plasma irradiation has been indicated as suitable for passivation of
$\mathrm{ZnO}$ radiative recombination centers giving visible emission (VIS) at $2.3 \mathrm{eV}$. However, a conventional heating technique in a $\mathrm{H}_{2}$ gas to dope hydrogen atom into $\mathrm{ZnO}$ causes the formation of defects such as oxygen vacancy or evaporation of $\mathrm{ZnO}$. Furthermore, $\mathrm{ZnO}$ was reduced through continuous irradiation with non-modulated hydrogen plasma.

Ohashi et al. applied Ar- $\mathrm{H}_{2}$ PMITP for this purpose [19, 20]. Use of the $\mathrm{ArH}_{2}$ PMITP provides less heat accumulation in the irradiation specimen than with the continuous plasma irradiation. Experimental conditions were as described below. They used on-time of $10 \mathrm{~ms}$ and off-time of $5 \mathrm{~ms}$. The rf frequency was $1 \mathrm{MHz}$, the input power level to a inverter power supply is $13 \mathrm{~kW}$ for a higher level, although it is $4 \mathrm{~kW}$ for a lower level. The hydrogen gas flow rate was set to $6 \times 10^{-3} \mathrm{~m}^{3} / \mathrm{min}$, whereas the Ar gas flow rate was 98 $\times 10^{-3} \mathrm{~m}^{3} / \mathrm{min}$. The total gas pressure was $27 \mathrm{kPa}$. The irradiation time was $300 \mathrm{~s}$. Details of the experimental setup are available in an earlier report [18].

Ohashi et al. confirmed in their experiments that $\mathrm{Ar}-\mathrm{H}_{2}$ PMITP irradiation does not reduce $\mathrm{ZnO}$, although a nonmodulated plasma irradiation does. Additionally, they found that improvement of photoluminescence of $\mathrm{ZnO}$ was achieved by irradiation of $\mathrm{ArH}_{2}$ PMITP. Fig. (21) shows the photoluminescence (PL) spectra of $\mathrm{ZnO}$. For original polycrystalline $\mathrm{ZnO}$, a broad VIS emission band was detected around $2.3 \mathrm{eV}$, and an ultraviolet (UV) emission band at $3.3 \mathrm{eV}$. After irradiation of $\mathrm{Ar}-\mathrm{H}_{2}$ PMITP, as portrayed in Fig. 21, the VIS emission band disappeared and the UV emission intensity increased by more than 10 times. Improvement in UV emission efficiency can be ascribed to the passivation of active centers, which are the origin of the VIS emission.

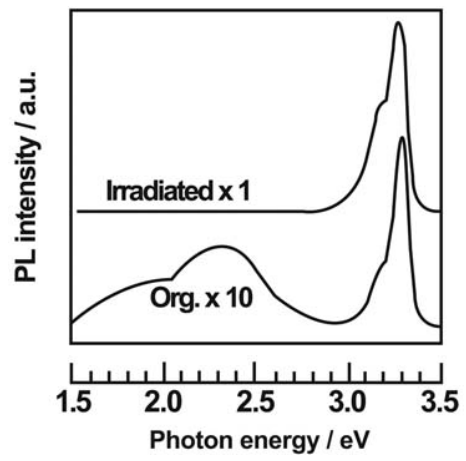

Fig. (21). Typical photoluminescence spectra of $\mathrm{ZnO}$ before and after hydrogen plasma irradiation for Polycrystalline ceramic [20].

\section{APPLICATIONS OF AR-O $\mathrm{O}_{2}$ PMITP FOR NANOPOWDER SYNTHESIS}

In the previous section, we found that heat flux and mass flux of atomic particles downstream of the plasma torch can be controlled by controlling the modulated coil current. This feature might be adopted for controlling nanoparticle synthesis if the modulated induction thermal plasma is used. We are now trying to apply the $\mathrm{Ar}-\mathrm{O}_{2}$ PMITP to $\mathrm{TiO}_{2}$ nanopowder synthesis to study the effect of current modulation on the synthesized nanopowder. This is the latest result on PMITP application. A plasma torch with the same configuration to that in the previous section was also used in this work. However, downstream of the torch, a vertical reaction chamber, a horizontal reaction chamber and a filter 
were installed for nanopowder synthesis. The $\mathrm{Ar} / \mathrm{O}_{2}$ gas flow rate was 90/10 slpm. Titanium powder with a mean diameter of $45 \mu \mathrm{m}$ was fed with Ar carrier gas with a water-cooled probe inserted from the top-center of the plasma torch to the plasma. The powder feed rate was set around 3.5-4.0 g/min. The pressure was fixed at 300 Torr. Injected raw titanium powder is vaporized in the thermal plasma, and $\mathrm{TiO}_{2}$ nanoparticles is nucleated from the titanium vapor and oxigen atom in the reaction chamber installed downstream of the plasma torch [6]. The time-averaged input power was fixed at $20 \mathrm{~kW}$, irrespective of that with and without coil current modulation. For PMITP, the on-time and the off-time in the coil current modulation was set to $12 / 3 \mathrm{~ms}$; the shimmer current level (SCL) is $65 \%$ in the modulation case, and $100 \%$ for the non-modulation case. Fig. (22) portrays the size distribution of synthesized particles by modulated induction plasmas at $\mathrm{SCL}=65 \%$ and $100 \%$. From this figure, results show that the particles synthesized by the PMITP at $\mathrm{SCL}=65 \%$ have a smaller mean-diameter and a smaller standard deviation than those by the non-modulated plasma at $\mathrm{SCL}=100 \%$.

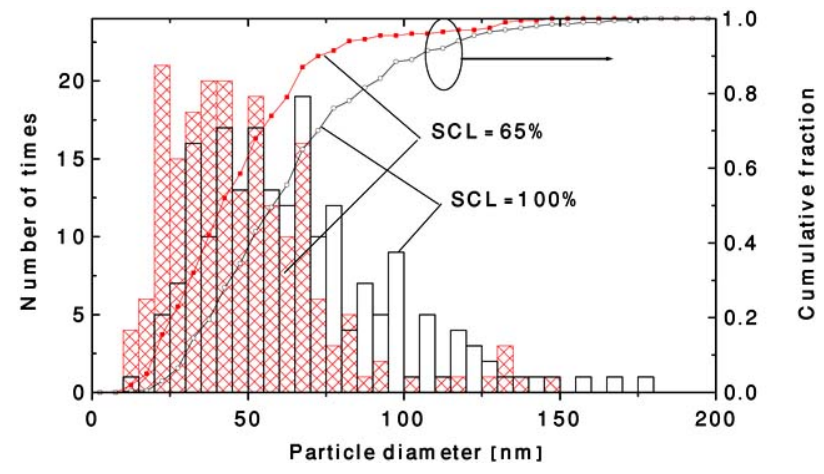

Fig. (22). Size distribution of $\mathrm{TiO}_{2}$ nanopowders synthesized by induction thermal plasma with and without current modulation.

\section{DEVELOPMENT OF ARBITRARY WAVEFORM MODULATED INDUCTION THERMAL PLASMA}

\subsection{Concept and Electric Circuit for AMITP}

Recently, we developed a new type of modulated induction thermal plasma system: it is a system of an
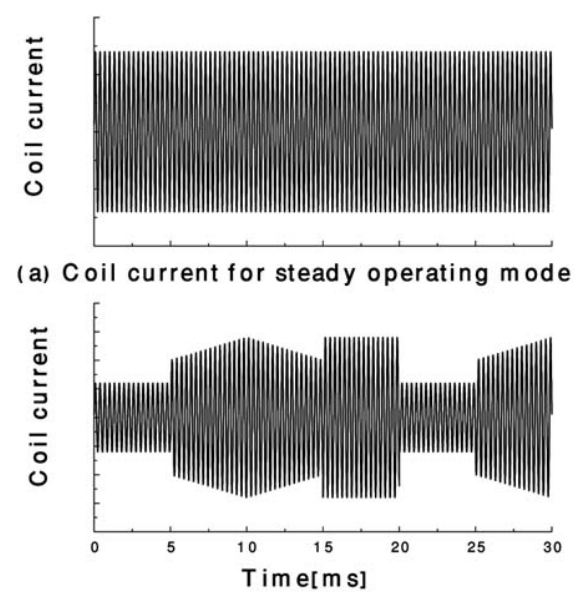

(b) Coil current for amplitude modulation

Fig. (23). Coil current for AMITP. arbitrary-waveform modulated induction thermal plasma (AMITP) [27]. This system can modulate the coil current sustaining an induction thermal plasma not only into a rectangular waveform but also into an externally determined waveform in milliseconds. Fig. (23) depicts an example of the coil current for AMITP. The coil current amplitude is modulated following an externally-given waveform, which is changed in milliseconds.

To realize AMITP, we have developed a new rf power supply which has IGBT and MOSFET elements. Fig. (24) shows the electric circuit for AMITP. The modulation in this case is achieved by switching IGBT. On the other hand, in this power supply, MOSFET elements are switched with a frequency to synchronize a frequency changed by a plasma load using phase-locked loop (PLL) control.

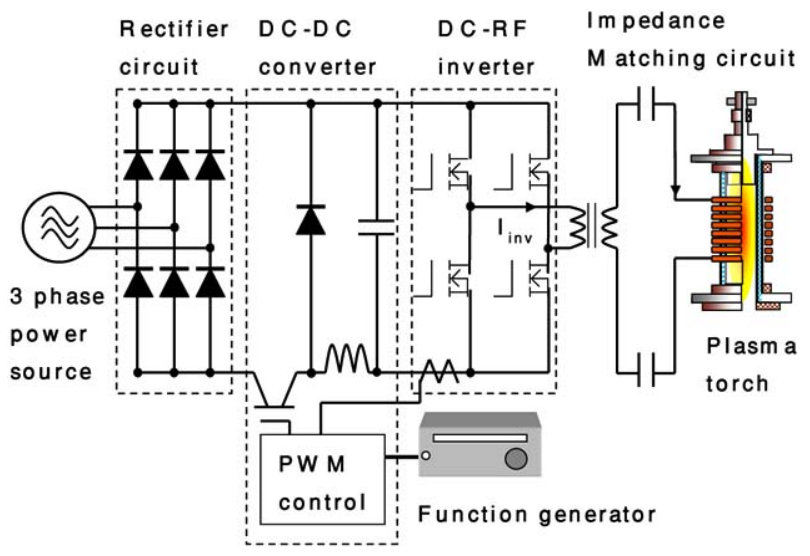

Fig. (24). Electric circuit for AMITP [27].

\subsection{Dynamic Behavior of Ar AMITP}

Fig. (25) shows (a) the modulation signal, (b) the inverter output current in root-meansquare value, (c) the radiation intensity of the Ar atomic line at $703 \mathrm{~nm}$, and (d) the Ar excitation temperature, in a triangular-rectangular waveform modulation case [27]. The input power is $10 \mathrm{~kW}$, and the pressure is $5.3 \mathrm{kPa}$ ( $=40$ Torr). The observation was conducted at $10 \mathrm{~mm}$ below the coil end. The observed radiation intensity changes periodically in a triangular waveform according to the modulation control signals with some delay compared to the modulation signal. The delay time was estimated roughly as $5 \mathrm{~ms}$ because of the time difference between the minimum of the coil current amplitude and the minimum of the radiation intensity. This delay time in the radiation intensity arises mainly from the thermal inertia of thermal plasmas, which is governed mainly by the mass density and specific heat of hightemperature Ar gas. Fig (25) (c) depicts the variation of the radiation intensity for the triangle-rectangular waveform. Results show that the radiation intensity for the rectangular part becomes higher than that for the first triangular part, which arises from the accumulated power inputted to the plasma.

It is interesting to see how the temperature changes following the modulation signal. The Ar excitation temperature was estimated using the two-line method with the two specified Ar lines at 703 and $714 \mathrm{~nm}$. Fig. (25) (d) shows the time evolution in the Ar excitation temperature in the case of triangular-rectangular waveform modulation [27]. 

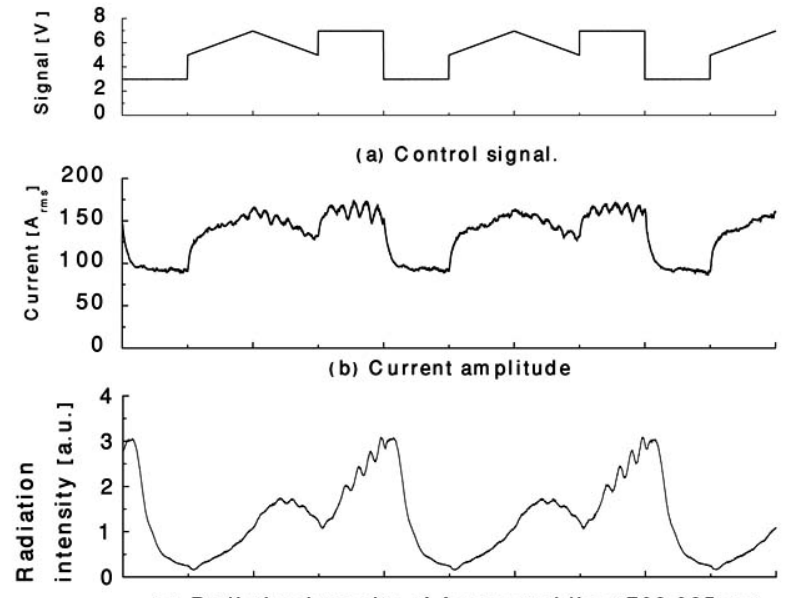

(c) Radiation intensity of A r spectral line $(703.025 \mathrm{~nm})$

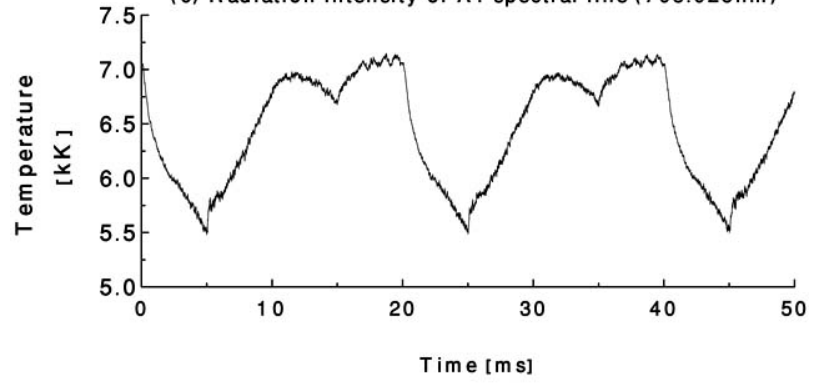

(d) A r excitation tem perature

Fig. (25). Time evolution in (a) control signal, (b) modulated coil current, (c) effective power, (d) load resistance, and (e) $\mathrm{Ar}$ excitation temperature measured in Ar AMITP [27].

The Ar excitation temperature is also modulated periodically following a triangular- rectangular control signal, changing from $5500 \mathrm{~K}$ to $7000 \mathrm{~K}$. These results imply that the Ar excitation temperature in Ar AMITP can be controlled using the modulated coil current in milliseconds in greater detail than that in PMITP.

\section{CONCLUSION}

This paper presented a review of recent results related to fundamental dynamic behaviors of PMITPs, in addition to applications of PMITPs. Results show that the modulation of the coil current can perturb high-pressure high-power thermal plasmas, and can control the temperature and radical densities. Especially, $\mathrm{Ar}$ excitation temperature in a molecular gas seeded Ar thermal plasma can be changed following the modulated coil current. Application of PMITP to surface modification processing is one candidate for use of chemically non-equilibrium effects occurring in PMITPs. Results also show that the coil current modulation can control the nanopowder size if the PMITP is used for nanopowder synthesis using thermal plasmas. In addition, a new type of modulated induction thermal plasma, AMITP, has been developed. Results demonstrate that more detailed control of the temperature is possible in AMITP than PMITP. The modulated thermal plasma is a promising radical and heat source for use in high-speed material processing.

\section{REFERENCES}

[1] Berghaus JO, Meunier JL, Gitzhofer F. Monitoring and control of $\mathrm{RF}$ thermal plasma diamond deposition via substrate biasing. Meas Sci Technol 2004; 15: 161-4.
Matsumoto S, Hino M, Kobayashi T. Synthesis of diamond films in an rf induction thermal plasma. Appl Phys Lett 1987; 51: 737-9.

Wang C, Inazaki A, Shirai T, et al. Effect of ambient gas and pressure on fullerene synthesis in induction thermal plasma. Thin Solid Films 2003; 425: 41-8.

Todorovic-Markovic B, Markovic Z, Mohai I, et al. Efficient synthesis of fullerenes in RF thermal plasma reactor. Chem Phys Lett 2003; 378: 434-9.

Girshick SL, Chiu CP, Muno R, et al. Thermal plasma synthesis of ultrafine iron particles. J Aerosol Sci 1993; 24: 367-82.

Ishigaki T, Oh SM, Li JG, Park DW. Controlling the synthesis of $\mathrm{TaC}$ nanopowders by injecting liquid precursor into RF induction plasma. Sci Technol Adv Mater 2005; 6: 111-8.

[7] Shigeta M, Watanabe T, Nishiyama H. Numerical investigation for nano-particle synthesis in an RF inductively coupled plasma. Thin Solid Films 2004; 457: 192-200.

[8] Huang H, Eguchi K, Yoshida T. Novel structured yttria-stabilized zirconia coatings fabricated by hybrid thermal plasma spraying. Sci Technol Adv Mater 2003; 4: 617-22.

[9] Chen WR, Wu X, Marple BR, Patnaik PC. Oxidation and crack nucleation/growth in an airplasma-sprayed thermal barrier coating with NiCrAlY bond coat. Surface Coatings Technol 2005; 197: 109-15.

[10] Ishigaki T, Xiaobao F, Sakuta T, Banjo T, Shibuya Y. Generation of pulse-modulated induction thermal plasma at atmospheric pressure. Appl Phys Lett 1997; 71: 3787-9.

[11] Sakuta T, Tanaka Y, Hashimoto Y, Katsuki M. Novel system of an inductively coupled thermal plasma with pulse amplitude modulation of electromagnetic field. Electr Eng Japan 2002; 138: 26-33.

[12] Sakuta T, Tanaka Y, Paul KC, Hossain MM, Ishigaki T. Nonequilibrium effects in pulse modulated induction thermal plasma for advanced processing. Trans Mater Res Soc Japan 2000; 25: 358.

[13] Tanaka Y, Sakuta T. Measurement of dynamic response time in pulse modulated thermal plasma. Trans Mater Res Soc Japan 2000; 25: 293-6.

[14] Hossain MM, Tanaka Y, Sakuta T. Transient nature of argon and molecular gas-seeded argon inductive thermal plasmas in pulse amplitude modulation approach. Trans IEE Jpn 2003; 123-PE: 1333-49.

[15] Hossain MM, Tanaka Y, Sakuta T. Dynamic responses of Ar- $\mathrm{CO}_{2}$ and $\mathrm{Ar}-\mathrm{N}_{2}$ induction thermal plasmas in pulse modulation approach: a numerical analysis. Thin Solid Films 2003; 435: 19-26.

[16] Tanaka Y, Sakuta T. Stable operation region and dynamic behavior of pulse modulated Ar thermal plasma with different molecular gases. Electr Eng Jpn 2003; 143: 1-11.

[17] Tanaka Y, Sakuta T. Temperature control of Ar induction thermal plasma with diatomic molecular gases by pulse-amplitude modulation of coil-current. Plasma Sources Sci Technol 2003; 12: 69-77.

[18] Ishigaki T, Haneda H, Okada N, Ito S. Surface modification of titanium oxide in pulse-modulated induction thermal plasma. Thin Solid Films 2001; 390: 20-5.

[19] Ohashi N, Ishigaki T, Okada N, Sekiguchi T, Sakaguchi I, Haneda $\mathrm{H}$. Effect of hydrogen doping on ultraviolet emission spectra of various types of $\mathrm{ZnO}$. Appl Phys Lett 2002; 80: 2869-71.

[20] Ohashi N, Ishigaki $\mathrm{T}$, Okada $\mathrm{N}$, et al. Passivation of active recombination centers in $\mathrm{ZnO}$ by hydrogen doping. J Appl Phys 2003; 93: 6386-92.

[21] Tanaka Y, Muroya T, Hayashi K, Uesugi Y. Simultaneous control of numerical enhancement of $\mathrm{N}$ atom and decrease in heat flux into reaction chamber using Ar- $\mathrm{N}_{2}$ pulse-modulated induction thermal plasmas. Appl Phys Lett 2006; 89: 031501.

[22] Tanaka Y, Muroya T, Hayashi K, Uesugi Y. Control of nitrogen atomic density and enthalpy flow into reaction chamber in $\mathrm{Ar}-\mathrm{N}_{2}$ pulse-modulated induction thermal plasmas. IEEE Trans Plasma Sci 2007; 35: 197-203.

[23] Tanaka Y, Hayashi K, Nakamura T, Uesugi Y. Influence of ontime on increased number density of excited nitrogen atom in pulse modulated induction thermal plasmas. J Phys D Appl Phys 2008; 41: 185-203.

[24] Tanaka Y, Sakuta T. Time-dependent two-dimensional chemical non-equilibrium modeling of $\mathrm{Ar}-\mathrm{N}_{2}$ pulse-modulated induction thermal plasma at atmospheric pressure for material processing. Trans Mater Res Soc Jpn 2004; 29: 3403-6. 
[25] Tanaka Y. Two-temperature chemically non-equilibrium modelling of high-power $\mathrm{Ar}-\mathrm{N}_{2}$ inductively coupled plasma at atmospheric pressure. J Phys D Appl Phys 2004; 37: 1190-205.

[26] Tanaka Y. Time-dependent two-temperature chemically nonequilibrium modelling of high-power $\mathrm{Ar}-\mathrm{N}_{2}$ pulse-modulated inductively coupled plasmas at atmospheric pressure. J Phys D Appl Phys 2006; 39: 307-19.

[27] Tanaka Y, Morishita Y, Okunaga K, Fushie S, Uesugi Y. Generation of high-power arbitrarywaveform modulated inductively coupled plasmas for materials processing. Appl Phys Lett 2007; 90: 071502

[28] Sakuta T, Ishigaki T. Non-equilibrium effects in pulse modulated induction thermal plasma for advanced material processing. Pure Appl Chem 1999; 71: 1845-52.

[29] Sakuta T, Tanaka Y, Paul KC, Hossain MM, Ishigaki T. Nonequilibrium effects in pulse modulated induction thermal plasma for advanced processing. Trans Mater Res Soc Jpn 2000; 25: 35-8.

(C) Schlickeiser et al.; Licensee Bentham Open.

This is an open access article licensed under the terms of the Creative Commons Attribution Non-Commercial License (http://creativecommons.org/licenses/by$\mathrm{nc} / 3.0 /$ ), which permits unrestricted, non-commercial use, distribution and reproduction in any medium, provided the work is properly cited. 